\title{
P04-11-20 Poster session
}

\section{The roles of RhoA/ROCK signaling pathway in myometrial contractions and cervical ripening in pregnant rats}

\author{
Robert Gaspar, Dora Domokos, Eszter Ducza, Judit Hajagos-Toth, Kalman F. Szucs \\ Department of Pharmacodynamics and Biopharmacy, University of Szeged, Hungary
}

Background: Activation of RhoA and Rho- associated kinases (ROCKs) is known to play a role in the regulation of smooth muscle contraction. There are few data on the RhoA and ROCKs expression levels in rat myometrium and cervix.

Aims: Our aim was to investigate the mRNA and protein expressions of RhoA and ROCKs in rat myometrial and cervices during pregnancy and parturition. Our further aim was to investigate the alteration of myometrial contractions and cervical resistance by RhoA and and ROCK inhibitors.

Methods: RhoA and ROCK I and II expressions were measured by real time PCR and Western blot analysis in nonpregnant and pregnant (5-22 days) rat uteri. The effects of ROCK (Y-27632, fasudil) and RhoA inhibitors (simvastatin) in 10-6 M concentration were measured in isolated organ bath experiments on myometrial and cervical samples.

Results: The myometrial mRNA and protein levels of RhoA decreased on the 5th day of pregnancy to day 22, then a sharp increase was detected at term. The mRNA and protein concentrations of ROCK I and II were down-regulated in the early stage of pregnancy, while it sharply increased during parturition. The cervical RhoA protein expression was decreased near and during parturition. ROCK I mRNA and protein expressions increased on day 22, while a pronounced decrease was measured during parturition. ROCK II mRNA and protein expressions were sharply reduced during parturition. The RhoA-inhibitor simvastatin relaxed the myometrial contractions, while increased the cervical resistance, although its effects were not followed by the alteration of RhoA expressions. The non-selective ROCK inhibitors fasudil and Y-27632 were inhibited the myometrial contractions and reduced cervical resistance.

Conclusions: The RhoA/ROCK signaling pathway has an impact on myometrial contractions and cervical ripening, especially in the final processes leading to delivery. ROCK inhibitors are potential drug candidates both for reducing preterm contractions or treating insufficient cervical ripening.

Funding: This work was supported by the Hungarian Scientific Research Fund (OTKA K116902). 